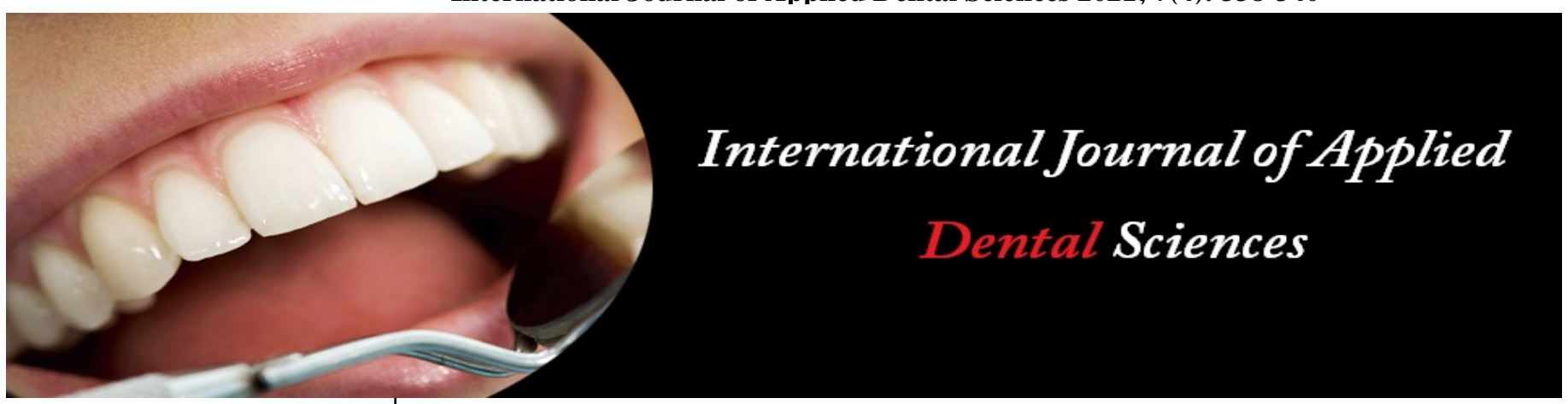

ISSN Print: 2394-7489

ISSN Online: 2394-7497

IJADS 2021; 7(4): 338-340

(C) 2022 IJADS

www.oraljournal.com

Received: 27-10-2021

Accepted: 21-12-2021

Dr. Sanjay Talnia

Assistant Professor, Department

of Dentistry, Adesh Medical

College and Hospital, Sahabad,

Haryana, India

Dr. Mamit Kumar

MDS, Senior Lecturer,

Department of Oral and

Maxillofacial Surgery, Maharishi

Markandeswar College of Dental

Science and Research Mullana,

Ambala, Haryana, India

Dr. Saurav Bhargava

MDS, Department of Oral and

Maxillofacial Surgery,

Department of Dentistry GTB

hospital Shahdra New Delhi,

India

Dr. Siddhant Sharma

MDS, Department of Oral and

Maxillofacial Surgery, Maharish

Markandeswar College of Dental

Science and Research Mullana,

Ambala, Haryana, India

Dr. Kartika Goswami

BDS, Maharishi Markandeswar

College of Dental Science and

Research Mullana, Ambala,

Haryana, India

Corresponding Author:

Dr. Mamit Kumar

MDS, Senior Lecturer,

Department of Oral and

Maxillofacial Surgery, Maharishi

Markandeswar College of Dental

Science and Research Mullana,

Ambala, Haryana, India

\section{Comparison of tramadol and Ketorol in management of pain after third molar surgery}

\author{
Dr. Sanjay Talnia, Dr. Mamit Kumar, Dr. Saurav Bhargava, Dr. \\ Siddhant Sharma and Dr. Kartika Goswami
}

DOI: $\underline{\text { https://doi.org/10.22271/oral.2021.v7.i4e.1400 }}$

\section{Abstract}

Aims: To evaluate the comparison of analgesic efficacy of preoperative intravenous (IV) ketorolac versus tramadol in preventing postoperative pain after mandibular third molar surgery.

Methodology: Hundred patients in the age group of 20-40 years with asymptomatic impacted mandibular molars were randomly allocated into one of the two groups. All the patients underwent third molar surgery under local anesthesia. Group I received IV ketorolac $30 \mathrm{mg}$ and Group II received IV tramadol $50 \mathrm{mg}$ preoperatively. The difference in postoperative pain was assessed by five primary parameters: pain intensity being measured hourly by Wong-Baker pain assessment scale for $6 \mathrm{~h}$, onset of analgesia, duration of action, total number of analgesics consumed, and patient's global assessment.

Results: Throughout the $6 \mathrm{~h}$ investigation period, patients reported significantly lower pain intensity scores, longer duration of action, lesser postoperative analgesic consumption, and better global assessment in ketorolac when compared to tramadol group. Patients in the ketorolac group significantly performed better than the tramadol group in terms of all parameters except onset of analgesia.

Conclusion: The result of the present study shows that preoperative IV ketorolac 30mg is more effective than tramadol 50mg for postoperative pain following third molar surgery.

Keywords: Ketorol, tramadol, third molar surgical extraction

\section{Introduction}

Swelling, trismus, and moderate to severe discomfort result with surgical removal of an impacted mandibular third tooth. After third molar surgery, better pain control may lead to a better recovery in terms of lifestyle and oral function. Ketorolac, tramadol, paracetamol, nalbuphine, and buprenorphine are some of the analgesics utilised in intravenous (IV) procedures ${ }^{[1]}$.

Ketorolac tromethamine, a member of the pyrrolo pyrrole group, has analgesic, antiinflammatory, and antipyretic effects and has been shown to be useful for postoperative dental pain. It can be administered intravenously, intramuscularly, or orally ${ }^{[2]}$.

Tramadol hydrochloride, a synthetic derivative of codeine, has been shown to be clinically useful in treating moderate to moderately severe pain while inducing minor respiratory depression and having a low risk for addiction ${ }^{[3]}$.

Since the pharmacologic and pharmacodynamic benefits of each of these medications have been reported. There is insufficient evidence to support the efficacy of one medicine over another in the management of analgesic effect after surgical removal of the third molar. The goal of this study was to assess the analgesic efficacy of IV ketorolac against tramadol in the treatment of pain following surgical removal of the third molar.

\section{Methodology}

A total of 100 patients were enrolled in the present study. 60 men and 40 women were recruited for the present study that visited for the third molar surgical extraction. Patients of both genders of age from 20 to 40 years and with the physical status ASA I were included in the study.

The Patients undergone clinical examination. The intraoral periapical radiographs, and orthopantomograms were recorded. Winter's WAR lines were drawn, and WHARFE assessment was done. 


\section{Inclusion criteria}

1. Only the, moderately difficult and very difficult (difficulty index of 5-7 and 7-10, respectively)

2. Patient aged 20 to 40

\section{Exclusion criteria}

1. History of allergic reactions to tramadol or ketorolac

2. Patient on nonsteroidal anti-inflammatory drugs (NSAIDs) within past 21 days

3. Acute pericoronitis

Ethical committee clearance was obtained for the study from the institutional board. Individuals who were eligible to participate explained about the study and signed informed consent obtained. Enrolled patients were distributed randomly into two groups:

Group I (ketorolac $30 \mathrm{mg}$ IV, ketorolac tromethamine) and Group II (tramadol $50 \mathrm{mg}$ IV, tramadol hydrochloride).

The randomization number was kept in an opaque, sealed envelope, and the patient coding was only accessible to the primary investigator. The medicines and groups were unknown to the patients, surgeon (assistant investigator), and statisticians. On the day of surgery, a lead investigator unsealed this sealed envelope holding the patient coding and the drug to be administered; as a result, the drug was placed into a 2cc syringe. In the datasheet, the assistant investigator noted all of the parameters. The triple blind study design was used in this investigation since all information about both medicines was kept hidden until the end of the study.

Loaded syringe containing either ketorolac $30 \mathrm{mg} / \mathrm{ml}$ or tramadol $50 \mathrm{mg} / \mathrm{ml}$ was administered into the cephalic vein in the antecubital fossa 15 min preoperatively. All the patients underwent extraction of third molars by the same surgeon and an assistant, under local anesthesia (2\% lignocaine with 1:200,000 adrenaline) for blocking inferior alveolar nerve and lingual and long buccal nerve. Surgical field was prepared and strict aseptic techniques were followed. Wards incision was given and tooth extraction was done by osteotomy and tooth sectioning, followed by socket toileting and wound closure using 3-0 mersilk suture.

Rescue oral analgesic (aceclofenac $100 \mathrm{mg}+$ paracetamol 500 $\mathrm{mg}+$ serratiopeptidase $10 \mathrm{mg}$ ), antibiotics, and antiemetics were prescribed, and postoperative instructions were advised. Following the surgery, participants were asked to describe the time when they first felt pain, at which point the Wong Baker pain assessment scale was used to conduct hourly pain assessments ${ }^{[4]}$. Patients were discharged and told to return for a regular checkup after the discomfort reduced and the immediate postoperative problems were ruled out. Patients were also requested to keep track of how many rescue analgesics they took every day up until the fifth postoperative day, and sutures were removed on the seventh day after good healing. A routine follow-up assessment included a look at any potential side effects from the study medicines.

\section{Statistical analysis}

It was done using SPSS software version 20.0 (IBM Corp. Armonk, NY. USA). Statistical data in relation to onset of action, duration of action, sum of pain intensity, total number of analgesics consumption during 5 postoperative days, and efficacy of analgesics postoperatively were interpreted by Student's t-test. A $\mathrm{P}<0.05$ was considered statistically significant.

\section{Results}

The present study included administration of IV ketorolac or tramadol in 100 patients each preoperative to third molar surgery. Both the drugs were compared for onset of analgesia, duration of action, sum of pain intensity scores for $6 \mathrm{~h}$, total number of analgesics consumed, and global assessment. The mean age of ketorolac and tramadol was 26 and 25 respectively which were not significant statistically. 40 females and 60 males were enrolled in the present study [Table 1].

Duration of action between the groups showed better results for ketorolac group (9.56 hours) compared to tramadol (4 hours). The onset of analgesia between group showed that tramadol was having better and faster onset as compared to ketorolac.

The sum of hourly pain intensity scores between the groups showed better results with ketorolac than tramadol. Patients with ketorolac group (3.01) had consumed less analgesics as compared to tramadol group (7.91). Patients were requested to grade the surgical procedure and pain relief status using global assessment scale, ketorolac group showed better results (2.01) than tramadol group (3.51) [Table 2]

Table 1: Demographic data of the study population

\begin{tabular}{|c|c|c|c|c|}
\hline Parameters & N (\%) & Ketorolac & Tramadol & P value \\
\hline \multicolumn{5}{|c|}{ Age } \\
\hline $20-30$ & 57 & $26.3 \pm 6.1$ & \multirow{2}{*}{$25.2 \pm 5.9$} & \multirow{2}{*}{0.322} \\
\hline $30-40$ & 43 & Sex \\
\hline \multicolumn{5}{|c|}{0.288} \\
\hline Female & 40 & 20 & 20 & \multirow{2}{*}{0.20} \\
\cline { 1 - 3 } Male & 60 & 30 & 30 &
\end{tabular}

$* \mathrm{P}$ value of less than 0.05 was considered statistically significant

Table 2: Comparison of various parameters related to efficacy of the drugs in the study population

\begin{tabular}{|c|c|c|c|}
\hline Parameters & Ketorol & Tramadol & P value \\
\hline Duration of action & $9.46 \pm 1.50$ & $4.0 \pm 1.43$ & $0.001 *$ \\
\hline Onset of analgesia & $14.40 \pm 3.06$ & $3.22 \pm 1.05$ & $0.001 *$ \\
\hline Sum of pain intensity score of 12 hours & $33.4 \pm 6.87$ & $53.22 \pm 4.45$ & $0.001 *$ \\
\hline Total number of analgesics consumed during 5 post- operative days & $3.01 \pm 2.23$ & $7.91 \pm 3.01$ & $0.001 *$ \\
\hline Global assessment & $2.01 \pm 1.12$ & $3.51 \pm 0.52$ & $0.001^{*}$ \\
\hline
\end{tabular}

*P value of less than 0.05 was considered statistically significant

\section{Discussion}

The development of pain hypersensitization may be decreased or eliminated by administering analgesics prior to the painful stimulation, resulting in less postoperative discomfort. 5 Third molar surgery is a common model for evaluating analgesic efficacy because it causes pain that is often consistent in degree, allowing for good differentiation between weak and strong analgesics. Ketorolac was developed as a parenteral nonsteroidal anti-inflammatory drug (NSAID) for the treatment of postoperative pain, and its analgesic effectiveness has been compared to that of morphine. Ketorolac suppositories are also more effective than diclofenac, according to comparative research ${ }^{[6]}$.

Tramadol is thought to have a multimodal activity, with an 
analgesic efficacy comparable to ketorolac. Any significant difference in pain between the two study groups in this study can thus be attributed to the medication effect. In a research comparing IV tramadol and ketorolac in terms of analgesic potency after third molar surgery, Ong et al. ${ }^{[7]}$ found that ketorolac had a longer analgesic duration than tramadol, with less rescue analgesic intake overall. This study's findings matched those of the current study.

Ong et al. ${ }^{[7]}$ measured postoperative pain following third molar surgery for 6 hours and found that individuals in the ketorolac group had considerably less pain than those in the tramadol group throughout the study period. Preoperative IV ketorolac provided better pain alleviation and lower pain intensity scores in our trial. Due to its peripheral modes of action, ketorolac proven to be a better medication for postoperative pain management following third molar surgery. Shaik et al. ${ }^{[8]}$ found that tramadol is an acceptable and safe analgesic for the management of post-extraction pain, and that it is more effective than ketorolac in terms of extended analgesia and minimum side effects. The discrepancy could be related to analgesics taken orally.

Vitterio Colletti et al. ${ }^{[9]}$ conducted a clinical trial to compare the postoperative analgesic effect and therapeutic tolerability of tramadol administered by IV injection when compared with that of ketorolac. During the 3 days of trial, the number of ampoules used did not differ significantly.

For tramadol, the mean was $1.4 \pm 0.1$ ampoules on the day of surgery, decreasing to $0.4 \pm 0.1$ ampoules on day 1 and $0.1 \pm$ 0.1 ampoules on day 2 postoperatively. For ketorolac, the mean number of ampoules was $1.5 \pm 0.1$ on the day of surgery, decreasing to $0.6 \pm 0.1$ on day 1 and $0.1 \pm 0.1$ on day 2. The overall number of analgesics consumed in this trial was shown to be lower in the ketorolac group than in the tramadol group. Because Group I has better analgesic efficacy for third molar surgical pain than Group II, it's possible that the pathogenesis of dental pain is largely inflammatory, and NSAIDs have been shown to provide excellent dental pain relief, despite the fact that Tramadol does not affect prostaglandin synthesis and has no anti-inflammatory effects. In their study, Bouloux GF et al. found that tramadol was a superior medication than ketorolac for postoperative pain control following nasal operations. 9 In a research comparing the analgesic efficacy of tramadol hydrochloride to diclofenac sodium in dentoalveolar surgery, Shah et al. The goal of this study was to develop a safe and effective analgesic alternative to NSAIDs for patients who couldn't take NSAIDs during dentoalveolar surgery. The two medications had similar analgesic efficacy, however tramadol outperformed diclofenac. Tramadol can be used for postoperative analgesia following dentoalveolar surgery, especially when NSAIDS are not recommended ${ }^{[10]}$.

Because of the type of pain following third molar operations, ketorolac performed better than tramadol in our study. The pathophysiology of dental pain differs from that of general surgical pain. Because dental pain is mostly inflammatory, nonsteroidal anti-inflammatory drugs (NSAIDs) are more effective than opioids in treating it. Discomfort and skin reactions at the injection site are the most prevalent side effects of parenteral ketorolac, but only five people in our study reported significant pain at the injection site, and none of them had local skin reactions.

\section{Conclusion}

Despite the fact that tramadol is an opioid, ketorolac provided greater pain management after third molar surgery. The nature of dental pain, which is predominantly inflammatory, may explain why it is best controlled with an NSAID rather than an opiate. Despite the small sample size, we conclude that ketorolac should be preferred over tramadol for the management of postoperative pain after surgical removal of impacted mandibular third molars. However, more research trials with a larger sample size and a longer follow-up period are required to confirm the findings.

\section{References}

1. Ceccheti MM, Negrato GV, Peres MP, Deboni MC, Naclério-Homem Mda G. Analgesic and adjuvant anesthetic effect of submucosal tramadol after mandibular third molar surgery. Oral Surg Oral Med Oral Pathol Oral Radiol. 2014;117:e249-54.

2. Snyder M, Shugars DA, White RP, Phillips C. Pain medication as an indicator of interference with lifestyle and oral function during recovery after third molar surgery. J Oral Maxillofac Surg. 2005;63:1130-7.

3. GaribaldiJA, Elder MF. Evaluation of ketorolac (Toradol) with varying amounts of codeine for postoperative extraction pain control. Int $\mathrm{J}$ Oral Maxillofac Surg. 2002;31:276-80.

4. Lanzetta A, Vizzardi M, Letiza G, Matorona U, Sanfillipo A, Osti L, et al. Intramuscular tramadol versus ketorolac in patients with orthopaedic and traumatologic postoperative pain. Curr Ther. Res 1998;59:39-46.

5. Gopalraju P, Lalitha RM, Prasad K, Ranganath K. Comparative study of intravenous tramadol versus ketorolac for preventing postoperative pain after third molar surgery-A prospective randomized study. J Craniomaxillofac Surg. 2014;42:629-33.

6. Tarkkila P, Tuominen M, Rosenberg PH. Intravenous ketorolac vs diclofenac for analgesia after maxillofacial surgery. Can J Anaesth. 1996;43:216-20.

7. Ong KS, Seymour RA, Chen FG, Ho VC. Preoperative ketorolac has a preemptive effect for postoperative third molar surgical pain. Int $\mathrm{J}$ Oral Maxillofac Surg. 2004;33:771-6.

8. Shaik MM, Kumar J, Mobina S, Satyanarayana N, Sunitha P. Comparative study of tramadol and ketorolac in the pain management of third molar tooth extraction. J Coll Med Sci. 2010;6:35-43.

9. Bouloux GF, Punnia-Moorthy A. Bupivacaine versus lidocaine for third molar surgery: A double-blind, randomized, crossover study. J Oral Maxillofac Surg. 1999;57:510-4.

10. Shah I, Zaeem K, Ibrahim MW, Hussain I, Hassan A. Comparison of analgesic efficacy of tramadol hydrochloride with diclofenac sodium in dento-alveolar surgery. Pak Oral Dent J. 2007;28:241-4. 